\title{
IDADE E CRESCIMENTO DA ABRÓTEA (Urophycis brasiliensis) CAPTURADA NO SUL DO BRASIL
}

\author{
ANDRADE, H.A.; DUARTE-PEREIRA, M. \& J.L. ABREU-SILVA \\ UNIVALI - CTTMar \\ humber.andrade@univali.br
}

\begin{abstract}
RESUMO
A abrótea (Urophycis brasilienis) é uma das principais espécies capturadas em arrastos de fundo na costa sul e sudeste do Brasil. Estimativas feitas durante o projeto REVIZEE indicam que o recurso pode estar sendo sobre-explorado. Entretanto, há uma insegurança quanto a esta avaliação, pois há incertezas quanto à determinação da idade. A redução dessas incertezas foi o objetivo desse trabalho. Foram analisados 390 otólitos de exemplares desembarcados para comercialização em 1997 e 1998, e uma amostra adicional de 50 otólitos de exemplares capturados em 2003 menores do que o tamanho comercial. A aplicação de dois métodos de validação reforçou a hipótese de que haja a formação de um anel por ano, definido por um par de bandas, uma opaca e uma translúcida. As idades mínimas e máximas observadas na captura comercial foram de 3 e 16 anos respectivamente. Os parâmetros do modelo de crescimento de von Bertalanffy foram estimados para os sexos agrupados e para as fêmeas separadamente, devido ao pequeno tamanho amostral do lote de dados de machos. Para fêmeas a equação estimada é $L t=75,67\left(1-\mathrm{e}^{-0,106(t+3,05)}\right)$, enquanto que para os sexos agrupados tem-se $L t=62,25\left(1-\mathrm{e}^{-0,106(t+0,53)}\right)$. Essas são as estimativas mais acuradas disponíveis no momento e devem ser utilizadas em futuras análises de avaliação de estoques.
\end{abstract}

Palavras Chave: Idade, Crescimento, Otólito, Urophycis brasilienis.

\section{AGE AND GROWTH OF THE BRAZILIAN CODLING (Urophycis brasiliensis) CAUGHT IN THE SOUTH OF BRAZIL}

\begin{abstract}
The Brazilian codling (Urophycis brasilienis) is one of the most important species usually caught by trawlers offshore of south and southwest Brazilian coast. Estimates gathered during the REVIZEE project indicated that this fishery resource is probably overexploited. Nevertheless, there is some doubt on the validity of this diagnosis due to uncertain on age determinations used for the stock assessment analysis. The scope of this work was to lessen this uncertainty. We analyzed 390 otoliths extracted from saleable fish landed in Itajaí-SC during 1997 and 1998, and 60 otoliths extracted from fish smaller than the saleable size, landed in 2003 . The results obtained by two validation methods support the hypothesis that one growth increment (i.e. couple of a translucid plus a opaque band) is layered each year. The youngest and the oldest fishes found were 3 and 16 years old respectively. We estimated parameters of the von Bertalanffy growth model for female data set and for both sexes pooled. The small sample size makes the growth estimation impractical for males. The female growth curve was $L t=75,67\left(1-\mathrm{e}^{-0,106(t+3,05)}\right)$, while the curve estimated for pooled data set was $L t=62,25\left(1-\mathrm{e}^{-0,106(t+0,53)}\right)$. These are the most accurate parameters for the Brazilian codling available and should be used in the future stock assessment analysis.
\end{abstract}

Keywords: Age, Growth, Otolith, Urophycis brasilienis. 


\section{INTRODUÇÃO}

A pescaria de recursos demersais realizada através de arrasto e emalhe de fundo gera capturas expressivas na costa sudeste-sul do Brasil (Kotas, 1991; GEP/UNIVALI, 2002). Em Santa Catarina cerca de $50 \%$ de toda a frota pesqueira é dedicada à captura de recursos demersais. Esta modalidade pesqueira é caracteristicamente multi-específica. Portanto, cerca de 15 categorias de pescado compõe a maioria da captura obtida (GEP/UNIVALI, 2002). Entre essas categorias, a abrótea tem destacada importância. Duas espécies são usualmente desembarcadas com essa sinonímia, a Urophycis brasiliensis e a U. mystaceus, também denominada de abrótea-de-profundidade. Enquanto essa última começou a receber atenção somente a partir do final da década de 1990 , a primeira (U. brasiliensis) é um recurso tradicionalmente explorado há muitas décadas pelas frotas de arrasto e emalhe de fundo. Apesar da pescaria da abrótea (termo para denominar somente a $\mathrm{U}$. brasiliensis de agora em diante) ser realizada principalmente no Brasil e no Uruguai, a espécie tem uma distribuição um pouco mais ampla, ocorrendo desde a costa sudeste do Brasil até o norte da Argentina, em profundidades que variam de 10 a $220 \mathrm{~m}$ (Cohen \& Inada, 1990; Haimovici et al., 1996).

A U. brasiliensis destaca-se pelo volume desembarcado, pelo preço de mercado e pela regularidade com que é capturada. Apesar da importância desse recurso, até então foram realizados poucos estudos sobre a dinâmica populacional da espécie, e portanto, há poucas informações biológicas que possam auxiliar na avaliação e na administração do estoque pesqueiro. Esse aparente descaso é fruto de uma concepção antiga de que a abrótea não é um dos alvos principais da pescaria. A algum tempo atrás o interesse econômico e científico esteve voltado para as espécies de maior valor individual (preço por quilo), tradicionalmente tidas como alvo (e.g. camarão-rosa). No entanto, à medida que os rendimentos (i.e. captura em peso) desses recursos tradicionais escasseou, cresceu a importância das espécies que originalmente não eram consideradas alvo de pesca. Atualmente as capturas de abrótea superam em muito as capturas de vários outros recursos tradicionais. Por exemplo, em 2003 foram desembarcadas em Santa Catarina cerca de 2.800 t de abrótea, $1.300 \mathrm{t}$ de linguados e somente $111 \mathrm{t}$ de camarão-rosa (GEP/UNIVALI, 2004). Não há dúvida que a abrótea é hoje um dos alvos principais das frotas que exploram recursos demersais. Portanto, há atualmente uma grande demanda por informações sobre a dinâmica populacional da abrótea (e.g. crescimento), que auxiliem na tomada de decisões de manejo.

Algumas estimativas preliminares feitas durante o Programa de Avaliação do Potencial Sustentável dos Recursos Vivos na Zona Econômica Exclusiva (REVIZEE) indicaram que o recurso pode estar sendo sobre-explorado no sentido de que o esforço de pesca atual é maior do que o que seria recomendável para que o rendimento fosse otimizado (Andrade et al., 2001). Entretanto, há uma grande insegurança quanto a esta avaliação do estoque em função das incertezas ainda existentes quanto às estimativas de idade da espécie obtidas a partir de leituras de otólitos. Andrade et al (2001) destacam que o nível elevado de discordância entre leitores e os resultados incertos quanto à periodicidade de formação dos anéis, lançam dúvidas sobre a confiabilidade das estimativas. Se há incertezas quanto as idades e o crescimento, os valores de biomassa obtidos a partir do uso dessas informações, têm utilidade limitada.

No estudo da idade e do crescimento de peixes, análises de deposições de anéis etários em otólitos (estrutura óssea de aposição do ouvido interno dos peixes), têm sido utilizadas com sucesso desde 1899 (Panella, 1971; Ricker, 1975). Essas análises estão calcadas no fato de que há a deposição de uma matriz óssea nos otólitos à medida que a idade do peixe aumenta. Em linhas gerais, a análise 
consiste na contagem de bandas opacas e translúcidas depositadas no otólito, e em uma investigação da ciclicidade temporal da deposição dessas bandas (validação) (Campana \& Jones, 1992; Campana, 2001). Usualmente a tentativa de validação é realizada a partir da análise da variação temporal da dominância de um ou outro tipo de borda (Geffen, 1992). Se os anéis (um par de bandas opaca e translúcida) são validados como índice da idade dos indivíduos, as leituras são então usadas para a estimativa da idade e de parâmetros de modelos de crescimento. Esse tipo de abordagem tem sido aplicada com relativo sucesso no estudo de espécies do gênero Urophycis (Clay \& Clay, 1991; Acuña \& Morales-Nin, 1997; Martins \& Haimovici, 2000). Porém a dificuldade de leitura e de validação dos anéis etários parece ser característica desse gênero (e.g. U. chuss - Dery, 1988; U. tenuis - Clay \& Clay, 1991, e Lang et al., 1996; U. mystaceus Martins \& Haimovici, 2000), e no caso da $U$. brasiliensis permanecem as incertezas na determinação da idade e do crescimento (Acuña \& Morales-Nin, 1997; Andrade et al., 2001).

O objetivo deste trabalho foi o de melhorar as estimativas pretéritas apresentadas por Andrade et al. (2001) e ainda não publicadas em meio científico adequado. Portanto, deu-se particular atenção aos pontos frágeis destacados pelos autores citados acima. Procurou-se a definição de um padrão de leitura mais claro, que implicasse em menor discordância entre leitores. No caso da validação, além da tradicional técnica da "variação percentual do tipo de borda" foi também empregado um outro método baseado na variação temporal do comprimento médio de cada classe etária (Vazzoler, 1982). Em adição a base de dados existente (otólitos preparados - ver Andrade et al., 2001) foi substancialmente expandida, com a inclusão de amostras de indivíduos juvenis até então não avaliados. O objetivo foi obter estimativas de idade mais consistentes, ou uma confirmação dos resultados apresentados em Andrade et al. (2001).

\section{MATERIAL E MÉTODOS}

\section{Amostragem e Preparação dos Otólitos}

Quase a totalidade da abrótea capturada no sudoeste e no sul do Brasil provém da atividade das frotas de arrasto demersal, composta por diversas modalidades de embarcações (e.g. arrasteiros simples, de parelhas, etc) (GEP/UNIVALI, 2002). As amostras dessas frotas analisadas nesse trabalho foram obtidas a partir de dois esquemas de coleta distintos. Um destinado à amostra da abrótea desembarcada para comercialização, e o outro destinado à coleta de indivíduos com tamanho fora dos padrões do mercado, usualmente descartados em alto mar.

As amostragens biológicas da captura comercial foram obtidas entre abril de 1997 e dezembro de 1998 em Itajaí-SC (265'S/ $48^{\circ} 40^{\prime} \mathrm{W}$ ), um dos principais portos pesqueiros do Brasil. Essas coletas foram realizadas no momento em que eram realizados os desembarques do pescado a ser comercializado. Um descrição do sistema amostral pode ser encontrada em Perez et al. (1998). Nessas coletas foi realizada uma amostragem estratificada, ou seja, foi coletado aproximadamente o mesmo número de indivíduos por classe de comprimento. Esses indivíduos foram então levados para o laboratório quando então eram medidos (comprimento total em centímetros) e pesados (peso eviscerado em gramas). Em adição os sexos foram identificados e os dois otólitos "sagitta" foram retirados. Em muitos casos a identificação do sexo se mostrou impossível, uma vez que a abrótea é eviscerada em alto mar. A identificação do sexo ficou condicionada à avaliação de resquícios de gônadas que permaneceram após a evisceração.

A amostragem de peixes abaixo do tamanho comercial foi realizada de uma maneira prática e barata. Em outubro de 2003 foi coletado o resíduo (organismos de pequeno tamanho) que ficou nas esteiras usadas no desembarque comercial de pescado (transbordo do 
barco para caminhões e/ou câmaras frigoríficas). Amostras aleatórias do resíduo de dois desembarques foram levadas para o laboratório. Os peixes foram então medidos e tiveram seus otólitos retirados. Os sexos não foram identificados e os peixes não foram pesados, uma vez que normalmente estavam bastante danificados. Ao total, considerando os dois esquemas amostrais, foram coletados 390 exemplares, 24 machos, 250 fêmeas e 116 de sexo indeterminado (Tabela 1 ).

Um dos otólitos de cada exemplar foi envolto em um bloco de resina poliéster translúcida. Posteriormente foram feitos cortes na altura do núcleo do otólito, com o uso de uma cortadeira metalográfica de baixa rotação (ISOMET-Bühler), para que fossem obtidas fatias de aproximadamente $0,6 \mathrm{~mm}$ de espessura. As fatias foram coladas em lâminas de microscopia com o adesivo transparente (Enthelan-Merck) e analisadas através de imagens obtidas a partir de uma lupa estereoscópica e de um sistema de análise de imagens.

\section{Definição do Padrão de Leitura}

O uso da luz transmitida e do aumento de 1,5 à 2 (conforme o tamanho do otólito) foi adotado como procedimento de visualização do corte para a definição de um padrão de leitura. Nesse caso, a imagem do otólito apresenta-se como uma sucessão de bandas opacas (escuras) e translúcidas (claras), contendo uma região central escura que corresponde ao núcleo. Um par formado por uma banda clara e uma escura corresponde a um anel etário. $\mathrm{O}$ núcleo foi considerado correspondente ao período larval da espécie e foi então desconsiderado (Figura 1). Para fins práticos a leitura foi realizada sempre no eixo indicado à esquerda na figura 1. Até chegar a um acordo sobre o padrão de leitura foram necessárias três baterias de leituras prévias (três leituras para cada um dos três leitores) de um lote de 50 otólitos. A legibilidade dos otólitos é baixa em virtude da
Tabela 1 - Número total de indivíduos amostrados conforme mês e sexo. A coluna "Indeterminados" contém o número de indivíduos para os quais não foi possível identificar o sexo.

\begin{tabular}{cccccc}
\hline \hline Ano & Mês & Machos & Fêmeas & Indeterminados & Total \\
\hline 1997 & 4 & 2 & 3 & 0 & 5 \\
& 7 & 5 & 20 & 0 & 23 \\
& 9 & 0 & 8 & 0 & 8 \\
& 12 & 0 & 7 & 0 & 7 \\
1998 & 1 & 0 & 22 & 1 & 23 \\
& 2 & 1 & 15 & 0 & 16 \\
& 3 & 0 & 18 & 1 & 19 \\
& 4 & 8 & 11 & 0 & 19 \\
& 5 & 2 & 13 & 9 & 24 \\
& 6 & 0 & 15 & 0 & 14 \\
& 7 & 0 & 11 & 7 & 19 \\
& 8 & 1 & 13 & 3 & 17 \\
& 9 & 1 & 19 & 0 & 20 \\
& 10 & 0 & 15 & 8 & 23 \\
& 11 & 4 & 29 & 4 & 37 \\
too3 & 11 & 0 & 0 & 50 & 50 \\
\hline \hline
\end{tabular}

presença de inúmeros "cheks" (falsos anéis) e de anéis fracos. Após a bateria de testes as discordâncias entre os leitores atingiu níveis considerados como os mínimos possíveis. O coeficiente de variação (CV) (Chang, 1982) foi usado como índice para análise do nível de concordância (ou discordância). Normalmente essas análises de concordância são denominadas de "verificação". Após terem sido alcançados valores de CV satisfatórios, os testes foram encerrados e um único leitor prosseguiu com a análise dos demais cortes.

\section{Validação}

Os métodos para a verificação da periodicidade da deposição de anéis (validação) são variados. Neste estudo foram utilizadas duas metodologias: (a) análise da variação percentual do tipo de borda, translúcida ou opaca; e (b) análise da variação temporal do comprimento médio de cada classe etária. Uma descrição detalhada dessas técnicas pode ser encontrada em Vazzoler (1982), Campana \& Jones (1992), Geffen (1992) \& Campana (2001). Portanto, segue abaixo somente um resumo de cada uma delas. 
A análise da variação temporal do tipo de borda é possivelmente a técnica de validação mais simples, e por isso a mais difundida. Para aplicação desta técnica os dados foram agrupados por trimestre, e as freqüências percentuais do tipo de borda foram então calculadas. Caso haja uma alternância clara ao longo do ano, como por exemplo um período com dominância de borda clara e outro com predominância de borda escura, isto implicaria na formação de um par de bandas (uma clara mais uma escura) por ano. Portanto cada par de bandas (denominado anel) representaria um ano de vida. Essa técnica tem sido freqüentemente usada, inclusive para a validação da idade de outras espécies do gênero Urophycis (Clay \& Clay, 1991; Martins \& Haimovici, 2000).

A análise da variação temporal do comprimento médio das classes etárias (segunda técnica de validação), foi realizada também a partir do agrupamento trimestral dos dados. Ao aplicar essa técnica o esperado é que haja uma tendência de que os comprimentos médios por classe de idade aumentem à medida que o tempo passa e a última banda na borda do otólito é continuamente depositada. Essa tendência de crescimento do comprimento dentro de uma dada classe etária é interrompida quando há o término de deposição de um anel, e o início de deposição do próximo anel. Nesse momento, um peixe que vinha crescendo com cinco anos de idade por exemplo, passaria a ter seis anéis e saltaria para a próxima classe etária. A classe de cinco anos ficaria composta novamente de indivíduos de menor porte, provenientes da classe de quatro anos de idade. Dessa forma uma interrupção de crescimento por ano implica na formação de um anel anual.

\section{Estimativas de Crescimento}

O modelo de von Bertalanffy (1938) é normalmente o mais utilizado para descrever o crescimento dos peixes, incluindo diversas espécies de abróteas (U. tenuis - Clay \& Clay, 1991; U. brasiliensis - Acuña \& Morales-Nin, 1997; U. mystaceus - Martins \& Haimovici,

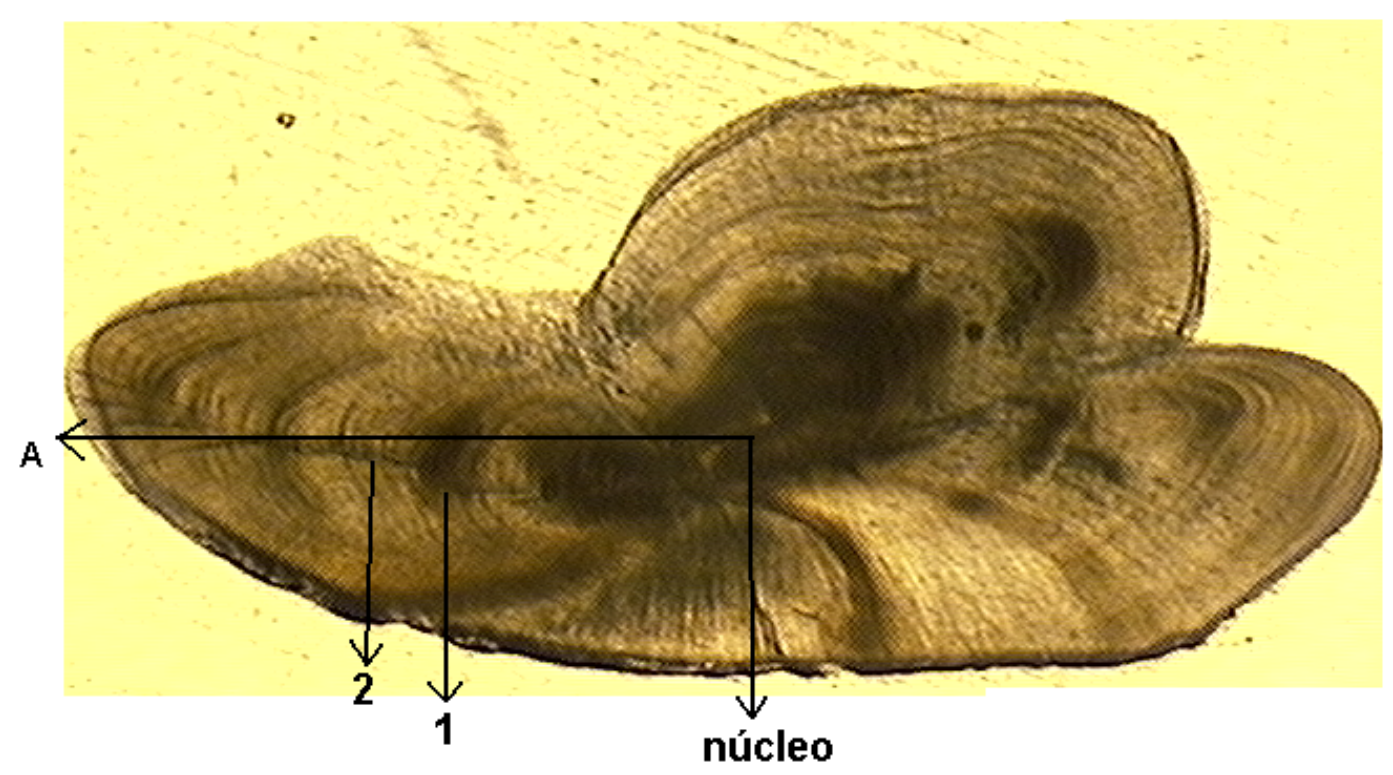

Figura 1 - Corte transversal de um otólito de abrótea (Urophycis brasiliensis) com indicações do núcleo, de uma banda translúcida (1), do início de uma opaca (2), e dos três eixos definidos para a leitura (A,B e C). 
2000). A equação de crescimento de von Bertalanffy (1938) é dada por

$$
L t=L \infty\left[1-e^{-k(t-t o)}\right]+\varepsilon
$$

onde L $t$ é o comprimento total em uma idade $t$ qualquer, $L \infty$ é o comprimento assintótico, $k$ é o coeficiente que representa a velocidade com a qual o comprimento do peixe se aproxima do comprimento assintótico e to é o intercepto da curva no eixo da variável independente (i.e. idade). $\varepsilon$ foi assumido como sendo um erro normal (média zero e variância $\sigma^{2}$ ). Os parâmetros do modelo (i.e. $L_{\infty}, k$ e to) foram estimados por iteração, pela minimização do logaritmo da verossimilhança.

\section{RESULTADOS}

\section{Distribuições de Freqüência de Comprimento}

A amostra foi mais representativa para os comprimentos compreendidos entre 30 e 40 $\mathrm{cm}$. No entanto, como esperado em virtude da amostragem estratificada, não há modas claramente predominantes acima do tamanho comercialmente aceito no mercado $(>20 \mathrm{~cm}$ ) (Figura 2). Exemplares grandes com mais de $50 \mathrm{~cm}$ representam apenas $5 \%$ da amostragem. No caso das amostras dos resíduos dos desembarques, os comprimentos entre 5 e $10 \mathrm{~cm}$ foram os dominantes.

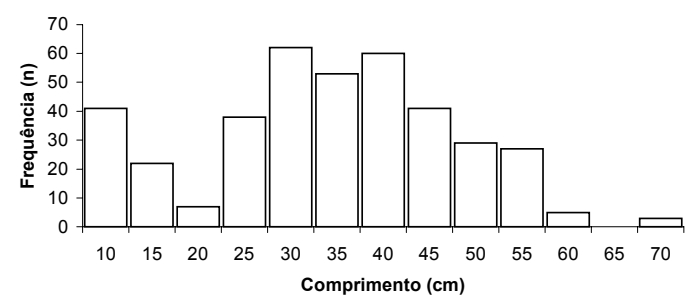

Figura 2 - Distribuição dos comprimentos observados na amostra.

\section{Verificação e Legibilidade dos Otólitos}

Na primeira sessão de leitura do lote de teste (50 otólitos), houve uma grande discordância entre os três leitores, sendo que os coeficientes de variabilidade (CV) superiores a 0,6 foram os dominantes. No entanto um aperfeiçoamento do padrão de leitura pode ser evidenciado pela sucessiva diminuição da discordância entre os leitores nas três séries subseqüentes de leitura (Figura 3). Note que não houve no entanto uma grande evolução a partir da segunda sessão. O CV médio foi de $0,72,0,29$ e 0,27 nas sessões de 1 a 3 respectivamente.

Tendências no geral similares foram observadas tanto com os dados agrupados por bimestre quanto por trimestre. No entanto o agrupamento bimestral (mais detalhado) resulta em problemas estatísticos em virtude do pequeno tamanho amostral em cada bimestre. Portanto optou-se pelo agrupamento trimestral. Os tipos de borda se alternaram uma vez quanto à dominância percentual por ano, indicando que é provável a formação de bandas opacas durante o inverno, e de translúcidas na primavera e no verão (Figura 4).

Quando aplicado o segundo método de validação, os comprimentos médios de cada

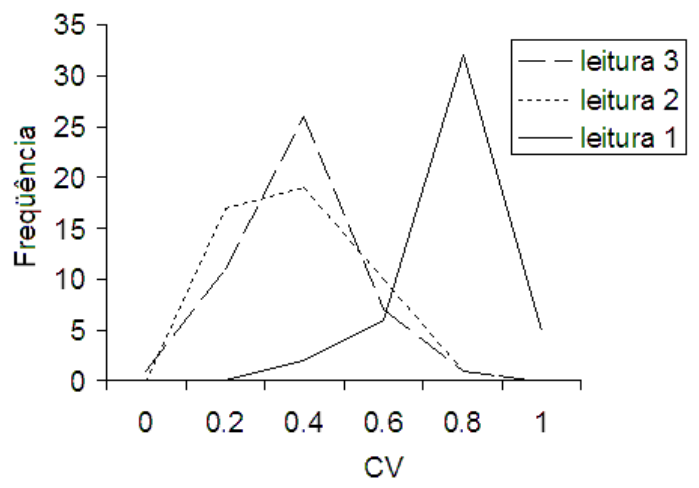

Figura 3 - Evolução dos valores do coeficiente de vulnerabilidade ao longo de três sessões de leituras realizadas com lotes de 50 otólitos. 
grupo de idade se mostraram altos no início do ano (verão) e decaíram no outono e no inverno, enquanto que uma tendência de incremento na primavera foi aparente na maioria dos casos (Figura 5). Novamente a formação de um anel anual aparece como a alternativa mais provável, visto que há uma clara quebra da continuidade do crescimento do comprimento médio de cada classe etária.

\section{Estimativas de Idade e de Parâmetros de Cres- cimento}

A menor idade para fêmeas nas amostras foi de 3 anos $(22,3 \mathrm{~cm})$ e a maior foi de 16 anos $(43,6 \mathrm{~cm})$. Para os machos as idade variaram de 4 anos $(24,3 \mathrm{~cm})$ a 11 anos $(51,5 \mathrm{~cm})$. Os exemplares da amostragem adicional feita com o rejeito de pesca, apresentaram idades entre 1 e 5 anos e comprimentos entre 7 e 26,5 $\mathrm{cm}$ respectivamente. Os maiores exemplares amostrados de machos e fêmeas tinham 51,5 e $57,9 \mathrm{~cm}$ respectivamente. No geral as idades entre 4 e 9 anos foram as dominantes nas amostras (Figura 6). Tanto machos como fêmeas apresentaram um padrão consistente de aumento de comprimento em função das idades observadas. A título de ilustração a curva ajustada aos dados agrupados é mostrada na figura 7.

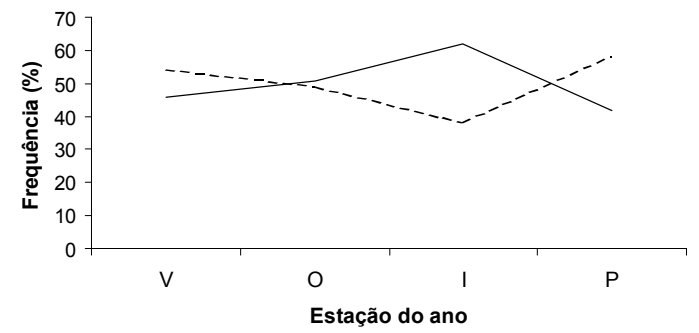

Figura 4 - Distribuição dos percentuais de bordas opacas (linha cheia) e translúcidas (linha pontilhada) ao longo das estações do ano: (V) verão, (O) outono, (I) inverno e (P) primavera. Dados de 202 otólitos de abrótea (Urophycis brasiliensis) provenientes de capturas realizadas na costa sudeste/sul do Brasil.
No processo de estimativa dos parâmetros de crescimento foi possível verificar novamente a evolução das estimativas de idade ao longo das três sessões iniciais de leituras realizadas. No caso do leitor definitivo (que realizou todas as demais leituras após a ses-

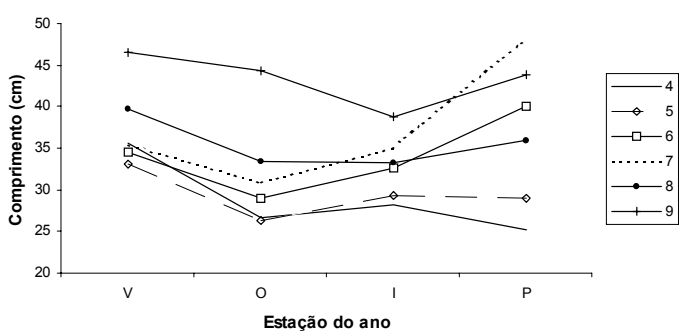

Figura 5 - Variação dos comprimentos médios $(\mathrm{cm})$ de diversas classes de idade (5 a 9 anos) de abrótea (Urophycis brasiliensis) no decorrer das estações do ano: $(V)$ verão, $(\mathrm{O})$ outono, $(\mathrm{I})$ inverno e $(\mathrm{P})$ primavera.

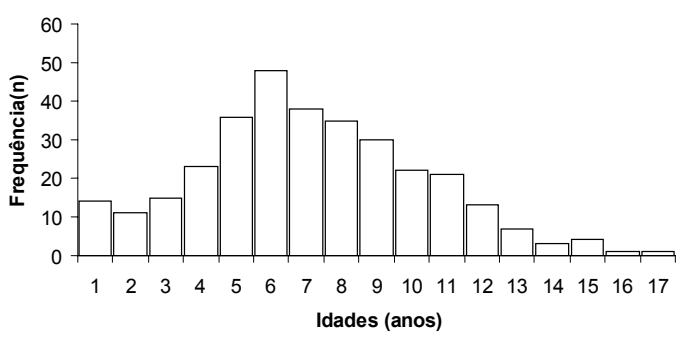

Figura 6 - Freqüência das idades observadas para a abrótea (Urophycis brasiliensis) $(\mathrm{n}=388)$, capturada na costa sudeste/sul do Brasil.

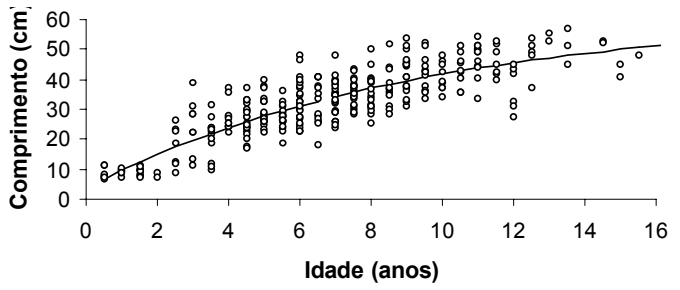

Figura 7 - Curva de crescimento para a abrótea (Urophycis brasiliensis) capturada na costa sudeste/ sul do Brasil. Os dados de ambos os sexos estão agrupados. 
são de testes), houve uma progressiva melhora das estimativas da primeira para a terceira e última sessão de testes (Tabela 2). Essa meIhora é evidenciada pelo aumento do coeficiente de determinação. Em adição as estimativas também se aproximaram de valores biologicamente mais realistas (e.g. o comprimento assintótico se aproxima dos maiores comprimentos observados nas amostras).

O modelo não foi ajustado aos dados de machos separadamente em virtude do pequeno tamanho amostral. No entanto, fica claro que ao agregar as amostras de machos, fêmeas e indivíduos de sexo indeterminado (lote composto majoritariamente pela amostra do resíduo), os parâmetros de crescimento se tornaram bem diferentes daqueles obtidos para as fêmeas separadamente.

\section{DISCUSSÃO}

Houve uma grande dificuldade na leitura das marcas de crescimento nos otólitos da abrótea costeira como já havia sido verificado para outras espécies congenéricas (e.g. Clay \& Clay, 1991) e em tentativas prévias de avaliação da própria U. brasiliensis (Andrade et al., 2001). Os otólitos apresentam diferentes formatos, inúmeros "checks" (falsos anéis) e as marcas de idade não ocorrem com espaçamento uniforme. A identificação dos anéis etários só foi possível com cortes transversais muito finos realizados na região central do otólito, enquanto que dados úteis só puderam ser obtidos após três sessões de leituras preliminares, intercaladas de discussões entre os leitores independentes. O monitoramento das variações dos coeficientes de variabilidade (CV) e das estimativas de crescimento provaram ser ferramentas objetivas adequadas na busca da definição de um padrão de leitura consistente dada a dificuldade de interpretação dos otólitos. O nível de concordância com esse procedimento foi maior do que o obtido por Andrade et al. (2001), e nesse sentido as leituras apresentadas aqui são mais confiáveis. No entanto, o grau de incerteza ainda é relativamente elevado como evidenciado pelos valores altos de CV.

Geralmente a banda translúcida é mais espessa e tende a ser a primeira a ocorrer após o núcleo. A banda opaca é normalmente estreita e é difícil a sua diferenciação quando ela está na borda dos cortes dos otólitos. Em conseqüência, a validação é também prejudicada. De fato, em ambos os métodos de validação utilizados a formação de um anel etário por ano aparece como a alternativa mais plausível, porém de forma não muito contundente. Contudo os resultados obtidos neste trabalho corroboram os obtidos por Andrade et al. (2001) e são também concordantes com estudos realizados com espécies do gênero Urophycis do Atlântico noroeste para as quais se estabeleceu uma periodicidade anual na formação dos anéis, com as bandas das bandas translúcidas sendo depositadas na primavera para (U. chuss -

Tabela 2 - Estimativas dos parâmetros de crescimento do modelo de von Bertalanffy para a abrótea (U. brasiliensis) capturado no sudeste e sul do Brasil. Na segunda coluna "A" indica os dados agrupados de machos, fêmeas e de indivíduos de sexo indeterminado, enquanto que "F" indica dados de fêmeas somente.

\begin{tabular}{ccccccc}
\hline \hline $\begin{array}{c}\text { Sessão de } \\
\text { Leitura }\end{array}$ & $\begin{array}{c}\text { Sexo } \\
\text { Tamanho da } \\
\text { Amostra }\end{array}$ & $L \infty$ & $k$ & $t o$ & $\mathrm{r}^{2}$ \\
\hline $1^{\circ}$ teste & $\mathrm{A}$ & 50 & 1377,72 & 0,002 & $-8,55$ & 0,49 \\
$3^{\circ}$ teste & $\mathrm{A}$ & 50 & 105,54 & 0,039 & $-2,79$ & 0,70 \\
definitiva & $\mathrm{A}$ & 320 & 61,30 & 0,108 & $-0,58$ & 0,71 \\
definitiva & $\mathrm{F}$ & 211 & 75,67 & 0,060 & $-3,05$ & 0,70 \\
\hline \hline
\end{tabular}


Dery, 1988). No único estudo realizado com alguma espécie do gênero Urophycis (i.e. U. mystaceus) no Atlântico Sul, houve indícios também pouco contundentes de que ocorre a formação de um anel anual (Martins \& Haimovici, 2000). Dado a sistemática indicação de ocorrência de um anel anual, consideramos que essa hipótese deve ser assumida para a validação, até que apareça algum resultado contraditório mais incisivo em algum estudo futuro. Sob essa prerrogativa, estaríamos portanto lidando com uma população de ciclo de vida relativamente longo, cujos indivíduos podem chegar a pouco menos de 20 anos de idade sob as atuais circunstâncias (i.e. taxas de mortalidade e recrutamento).

Com a amostragem realizada com exemplares abaixo do tamanho comercial foi possível caracterizar o início do desenvolvimento da espécie, de zero a quatro anos. Essa iniciativa de amostrar o resíduo das esteiras nas indústrias de pescado, pode ser importante para estudo do início da fase de crescimento das espécies capturadas comercialmente, visto que é freqüentemente difícil a coleta de exemplares juvenis nestes casos. No nosso caso, em algumas estimativas prévias de parâmetros de crescimento, em que não foram considerados os indivíduos abaixo do tamanho comercial, foram obtidos valores pouco realistas (i.e. e muito reduzidos e extremamente elevado). Claramente dados de indivíduos de pequena idade e tamanho são vitais para que estimativas de crescimento razoáveis possam ser obtidas. Portanto, recomenda-se que esse sistema de coleta do resíduo seja empregado mais freqüentemente em estudos de idade e crescimento.

Pelo menos três hipóteses poderiam ser consideradas para explicar o pequeno número de machos nas amostras da captura comercial: (a) machos (principalmente os de maior comprimento) não ocupam a área tradicionalmente explorada, ou emigram dela nos períodos de pescaria mais intensa, e não estão portanto muito vulneráveis à frota pesqueira; (b) as gônadas dos machos são mais frágeis que as das fêmeas, e nenhum resquício de tecido reprodutivo permanece após o peixe ser eviscerado a bordo; e (c) machos atingem comprimentos muito menores que as fêmeas (crescimento claramente diferenciado entre sexos). Há alguma evidência de que há migrações diferenciadas por sexo no caso de algumas espécies congenéricas (Dery, 1988; Clay \& Clay, 1991), e de que há distinção entre o padrão de distribuição espaço-temporal de machos e fêmeas da $U$. brasiliensis na costa do Uruguai (Acuña et al., 2000). Portanto, a primeira hipótese tem a princípio alguma credibilidade. No entanto, não há qualquer evidência mais contundente que indique que as demais hipóteses devem ser descartadas. Dessa forma, a razão da dominância das fêmeas nas capturas comerciais deveria ser investigada no futuro.

Apesar da amostragem realizada ter sido estratificada, e portanto não ser adequada para a avaliação da estrutura de comprimento e idade da captura, uma conclusão genérica pode ser feita a esse respeito. Independentemente do sexo, as idades de 5 a 9 anos, com comprimentos médios de cerca de $32 \mathrm{~cm}$, são as mais afetadas pela pescaria comercial.

Os parâmetros de crescimento obtidos com a inclusão de machos e de indivíduos de sexo indeterminado na amostra são muito diferentes daqueles obtidos para as fêmeas separadamente. Não há como precisar se a alteração dos parâmetros é decorrente de que há crescimentos diferenciados de machos e fêmeas, ou é fruto da inclusão de indivíduos de pequeno porte, de sexo indeterminado. Esse último fator certamente teve algum efeito, pois o to estimado para as fêmeas isoladamente foi muito diferente de zero, o que é um indício óbvio da falta de dados de peixes com comprimento e idade reduzida. No entanto, não há como descartar que haja um crescimento diferenciado por sexo antes da obtenção de uma estimativa de crescimento razoável para os machos separadamente. Essa problemática merece maiores atenções em estudos futuros. 
Finalizando, ressalta-se que as estimativas de parâmetros de crescimento que obtivemos são baseadas em leituras mais precisas (CV's menores), mas são similares às apresentadas em Andrade et al. (2001). No momento, ambas as estimativas poderiam ser igualmente aproveitadas em futuras avaliações de estoque.

\section{REFERÊNCIAS}

Acuña, A. \& B. Morales-Nin. 1997. Estudio sobre el uso del Sagitta para la Determinación de la Edad de la Brótola (Urophycis brasiliensis) (Pisces: Gaddidae). Anais do VII COLACMAR. 2226 set. Santos. 1:4-6.

Andrade, H. A; Lucato, S. H. B. \& G. S. Soares. 2001. Dinâmica populacional da abrótea (Urophycis brasiliensis) capturada no sul do Brasil. Relatório do programa REVIZEE, MMA-Brasília. $32 \mathrm{pp}$.

Campana, S. E. \& C. M. Jones. 1992. Analysis of Otolith Microstructure Data. In: D. K. Stevenson \& S. E. Campana. Otolith Microstructure Examination and Analysis. Can. Sp. Fish. Aquat. Sci. 117: 73-100.

Campana, S. E. 2001. Accuracy, precision and quality control in age determination, including a review of the use and abuse of age validation methods. J. Fish Biol. 59: 197-242.

Clay, D. \& H. Clay. 1991. Determination of Age and Growth of White Hake (Urophycis tenuis Mitchill) from the Southern Gulf of St. Laurence, Canada (including techniques for commercial sampling). Can. Tech. Rep. Fish. Aquat. Sci. 1828: $29+v i p$.

Chang, W. Y. B. 1982. A statistical method for evaluating the reproducibility of age determination. Can. J. Fish. Aquat. Sci. 39: 1208-1210.

Cohen, D. M. \& T. Inada. 1990. Gadiform fishes of the world (order Gadiformes). An annotaded and illustrated catalogue of cods, hakes, grenadiers and other gadiform fishes knowm to date. FAO species catalogue. $452 p$.

Dery, L. M. 1988. Red Hake, Urophycis chuss. In: Penttila, J. and Dery, L. M. Age Determination Methods for Northwest Atlantic Species. NOAA Technical Report NMFS. 72:49-57.

Geffen, A. J. 1992. Validation of Otolith Increment Deposition Rate. In: D. K. Stevenson \& S. E. Campana. Otolith Microstructure Examination and Analysis. Can. Sp. Fish. Aquat. Sci. 117: 101-113.

GEP/UNIVALI. 2002. Boletim Estatístico da Pesca Industrial de Santa Catarina - Ano 2001. Itajaí.

GEP/UNIVALI. 2004. Boletim Estatístico da Pesca Industrial de Santa Catarina - Ano 2003. Itajaí.

Haimovici, M; Martins, A. S. \& P. C. Vieira. 1996. Distribuição e Abundância de Peixes Teleósteos Demersais sobre a Plataforma Continental Sul do Brasil. Rev. Brasil. Biol. 56(1): 27-50.

Kotas, J. E. 1991. "By-Catch" de CamarãoRosa e Pescaria em Santa Catarina. Coleção Meio Ambiente Série Estudos Pesca. IBAMA/MMA: Brasília.

Lang, K. L.; Almeida, A. F. P.; Bolz, G. R. \& M. P. Fahay. 1996. The use of otolith microstructure in resolving issues of first year growth and spawning seasonally of White hake, Urophycis tenuis, in the Gulf of Maine - Georges Bank region. Fish. Bull. 112(1): 170-175.

Martins, R. S. \& M. Haimovici. 2000. Determinação de idade, crescimento e longevidade da abrótea de profundidade, Urophycis cirrata, Goode \& Bean, 1896, (Teleostei: Phycidae) no extremo sul do Brasil. Atlântica. 22: 57-70.

Panella, G. 1971. Fish Otoliths: Daily Growth Layers and Periodical Patterns. Science. 173:1124-1127.

Perez, J. A. A.; Lucato, S. H. B.; Andrade, H. A.; Pezzuto, P. R. \& M. Rodrigues-Ribeiro. 
NOTAS TÉC. FACIMAR, 8: 107-117, 2004.

1998. Programa de amostragem da pesca industrial desenvolvido para o porto de Itajaí, SC. Not. Téc. da FACIMAR 2: 93-108.

Ricker, W. E. 1975. Computation and Interpretation of Biological Statistic of Fish Populations. Bull. Fish. Res. Board Can. 191:1-382.
Vazzoler, A. E. A. M. 1982. Manual de métodos para estudos biológicos de populações de peixes - Reprodução e Crescimento. CNPq: Brasília

von Bertalanffy, L. 1938. A quantitative theory of organic growth (Inquiries on growth laws II). Human Biol. 10: 181-213. 